\title{
IMPACTO DE LA IMPLEMENTACIÓN DE UN ALGORITMO EN EL TRATAMIENTO DE NIÑOS HOSPITALIZADOS POR ASMA AGUDO
}

\section{IMPACT OF THE IMPLEMENTATION OF AN ALGORITHM IN THE TREATMENT OF CHILDREN HOSPITALIZED BY ACUTE ASTHMA}

\author{
Dra. Niscka Babaic M'; Dra. Daniela Parga A'; Dr. Francisco Prado A',2; Dr. Carlos Valdebenito P1,3; \\ Dr. Andrés Koppmann A. ${ }^{1,2}$; Int. Natalia Ríos G4. \\ 1 Residente de pediatría, Hospital Clínico San Borja Arriarán. Santiago, Chile. \\ 2 Pediatra Broncopulmonar, Hospital Clínico San Borja Arriarán. Departamento de Pediatría y Cirugía Infantil, Campus Centro, \\ Universidad de Chile. Santiago, Chile. \\ 3 Departamento de Pediatría y Cirugía Infantil, Campus Centro, Universidad de Chile Santiago, Chile. \\ 4 Interna de Medicina. Departamento de Pediatría y Cirugía Infantil, Campus Centro, Universidad de Chile. Santiago, Chile.
}

\begin{abstract}
Patients hospitalized for acute asthma treated with a pre-established algorithm could decrease hospital stay and critical bed (PICU) requirement. The objective of this article was to implement and evaluate the impact of a pre-established algorithm to treat children hospitalized for acute asthma. It is a cross-sectional and comparative study, with a prospective sample for convenience, of asthmatic children between 5 and 15 years admitted during 2017 without response to the first line of treatment in the emergency department. Patients with cardiorespiratory comorbidities and with direct admission to PICU were excluded. An algorithm was applied for 2 hours and its effectiveness was evaluated by a clinical score (PAS: English Pediatric Asthma Score). 55 patients were admitted, mean age 8.02 years, $41.8 \%$ female. The PAS decreased from 8 to 5 points at the end of the algorithm $(p<0.001)$. When comparing the results obtained with the group treated the previous year, without algorithm application in 51 patients with similar demographic characteristics, a shorter hospitalization was observed $(0.6$ days versus 0.95 days $(p<0.0368)$ ). The algorithm in acute asthma unified treatment criteria and times in its application. A rapid decrease in clinical score and a shorter hospital stay were observed.
\end{abstract}

Key words: acute asthma; algorithm; clinical effectiveness.

RESUMEN

Los pacientes hospitalizados por asma agudo tratados con un algoritmo preestablecido, podrían disminuir la estancia hospitalaria y requerimiento de cama crítica (UCIP). El objetivo de este trabajo fue el de implementar y evaluar el impacto de un algoritmo preestablecido para tratar a niños hospitalizados por asma aguda. Es un estudio transversal y comparativo, con una muestra prospectiva por conveniencia, de niños asmáticos entre 5 y 15 años ingresados durante el 2017 sin respuesta a la primera línea de tratamiento en el servicio de urgencia. Se excluyeron pacientes con comorbilidades cardiorespiratorias y con ingreso directo a UCIP. Se aplicó un algoritmo durante 2 horas evaluando su efectividad mediante puntaje clínico (PAS, por su sigla en inglés Pediatric Asthma Score). Ingresaron 55 pacientes, edad media 8,02 años, 41,8\% sexo femenino. El PAS disminuyó de 8 a 5 puntos al finalizar algoritmo $(p<0,001)$. Al comparar los resultados obtenidos con el grupo tratado el año anterior, sin aplicación de algoritmo en 51 pacientes con similares características demográficas, se observó una hospitalización más breve $(0,6$ días versus 0,95 días $(p<0,0368))$. El algoritmo en asma aguda unificó criterios de tratamiento y los tiempos en su aplicación. Se observó una rápida disminución del puntaje clínico y menor estancia hospitalaria. Palabras clave: asma agudo; algoritmo; efectividad clínica.

\section{INTRODUCCIÓN}

El asma afecta a 334 millones de personas en el mundo y es la enfermedad inflamatoria obstructiva crónica de la vía aérea más común en los niños (1). En Chile la prevalencia es elevada (2) y diferente según la edad, entre un 9 a $16 \%$ en niños de 6 a 7 años, y de un 7 a $12 \%$ en niños de 13 a 14 años $(2,3)$, con epidemiología similar a la observada en escolares de países desarrollados (3).

Las exacerbaciones de asma son habituales en los

\section{Correspondencia:}

Dr. Francisco Prado Atlagic

fpradoatlagic@gmail.com niños con una frecuencia cercana al $5 \%$ de las consultas en los servicios de urgencia, alcanzando un máximo de 10 a $15 \%$ en ciertas épocas del año, requiriendo hospitalización alrededor de $15 \%(4,5)$. Se estima que $10 \%$ de los pacientes asmáticos hospitalizados requieren ingreso a una unidad de cuidados intensivos, con una mortalidad de un $4 \%$ en aquellos que requieren ventilación mecánica invasiva (6).

El tratamiento del asma agudo está bien establecido e incluye un manejo de primera línea con broncodilatadores y corticoesteroides sistémicos (7). En el asma agudo moderado y severo la asociación de beta 2 de acción corta con bromuro de ipatropio (BI), utilizado en los servicios de urgencia en dosis repetidas, ha demostrado efectividad en reducir las tasas de hospitalización (8) y en disminuir la necesidad de 
traslado a unidades de mayor complejidad (9).

Pese a la existencia de puntajes clínicos que permiten determinar la severidad del asma agudo y definir la terapia a utilizar en cada caso (10), no existe un claro consenso respecto a los protocolos recomendados, que combinen los pilares farmacológicos antes mencionados con terapias de segunda línea como es el sulfato de magnesio (11) y terapias no farmacológicas destinadas a tratar la insuficiencia respiratoria aguda como son la oxigenoterapia de alto flujo (12) y la asistencia ventilatoria no invasiva (AVNI) (13).

El objetivo de este estudio fue describir y evaluar el impacto de un algoritmo de tratamiento en niños hospitalizados por crisis de asma refractaria al tratamiento de primera línea en el servicio de urgencia.

\section{PACIENTES Y MÉTODOS}

Se realizó un estudio transversal y comparativo con muestreo consecutivo por conveniencia de niños de 5 a 15 años, ingresados por asma agudo al servicio de pediatría del Hospital Clínico San Borja Arriaran (HCSBA), Santiago, Chile, entre diciembre del 2016 a diciembre del 2017. El grupo con el que se comparó los resultados fue una cohorte de pacientes del HCSBA evaluada en un estudio observacional prospectivo multicéntrico de pacientes hospitalizados por asma agudo realizado previamente durante el año 2016 (14). En este grupo no se utilizó el algoritmo para la toma de decisiones, y sólo se utilizó AVNI, dado que no se contaba con la incorporación sistemática de la OTAF. El sulfato de magnesio endovenoso se indicó en etapas posteriores de la evolución de la crisis, una vez ingresados a intermedio y en AVNI. Como terapia broncodilatadora para el paciente en AVNI, no se contaba con nebulizador de malla, por lo que se utilizó NBZ continua con un nebulizador jet Hudson o dosis repetidas de inhalador en IDM.

Se definió como asma agudo o exacerbación asmática a un episodio brusco y progresivo de obstrucción bronquial, de severidad variable, manifestado por opresión torácica, falta de aire, tos, sibilancias, disnea, polipnea y retracción. Esta definición clínica se relaciona con una disminución de los flujos espiratorios, que en pediatría rara vez son evaluados con flujometría, a pesar de la recomendación de las guías clínicas GINA (15). Se definió estado asmático a un episodio de asma agudo severo, refractario al tratamiento, con necesidad de hospitalizar y con insuficiencia respiratoria aguda con potencial requerimiento de soporte ventilatorio $(7,16)$.

Dada la dificultad para realizar flujometría en niños con crisis de asma, y como se ha demostrado una buena correlación con un puntaje clínico (17), se decidió usar el Score de asma pediátrico ((PAS) por su sigla en inglés; Pediatric Asthma Score) para evaluar la severidad y respuesta al tratamiento (10) (Tabla 1). La crisis de asma se categorizó

Tabla 1. Puntaje de severidad del asma agudo. Pediatric Asthma Score (PAS).

\begin{tabular}{|c|c|c|c|}
\hline Puntaje & 1 & 2 & 3 \\
\hline \multicolumn{4}{|l|}{ FR } \\
\hline 2 a 3 años & $<35$ & $35-39$ & $>40$ \\
\hline 4 a 5 años & $<31$ & $31-35$ & $>36$ \\
\hline 6 a 12 años & $<27$ & $27-30$ & $>30$ \\
\hline$>12$ años & $<24$ & $24-27$ & $>28$ \\
\hline $\mathrm{SpO2}$ & $>95 \% *$ & $90-95 \% *$ & $<90 \% * *$ \\
\hline Retracción costal & No o sólo intercostal & $\begin{array}{l}\text { Intercostal } \\
\text { y subcostal }\end{array}$ & universal \\
\hline Trabajo respiratorio (Disnea) & Habla con frases & Frases entrecortadas & Sólo palabras \\
\hline Auscultación & Espiración prolongada & $\begin{array}{l}\text { Sibilancias } \\
\text { espiratorias }\end{array}$ & $\begin{array}{c}\text { Sibilancias en } 2 \\
\text { tiempos }\end{array}$ \\
\hline
\end{tabular}

Se evalúan 5 características clínicas, a las que se les asigna un puntaje de 1 a 3 según severidad. Rango total del puntaje: 5-15. Puntaje < 8 (5-7): leve; 8 - 11: moderado; > 11 (12-15) severo.

Sp02: Saturación de pulso arterial de oxígeno. Fi02: Fracción inspirada de oxígeno.

FR: frecuencia respiratoria. * saturación con aire ambiental. ${ }^{* *}$ saturación con aire ambiental 0 el requerimiento de 02 para saturar sobre $93 \%$. 
Figura 1. Flujograma para tratamiento asma agudo severo.

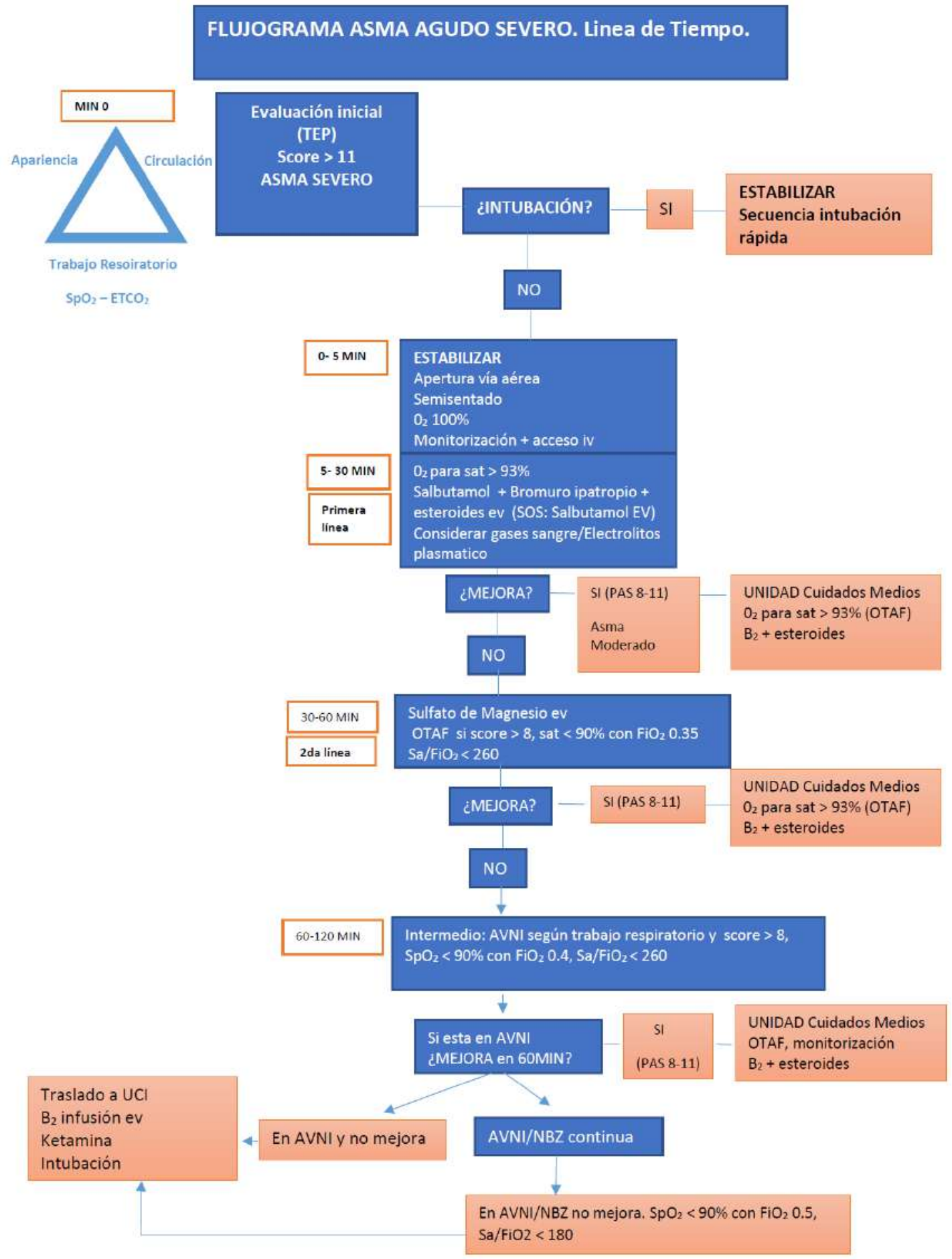

TEP: triángulo de evaluación pediátrica. Sp02: Saturación de oxígeno. ETC02: C02 al final de la espiración. OTAF: oxígeno termohumidificado de alto flujo. AVNI: asistencia ventilatoria no invasiva. Fi02: fracción inspirada de oxígeno. NBZ: nebulización. Sat: saturación. Iv: intravenoso. 
Tabla 2. Presentación y dosis de fármacos utilizados.

\begin{tabular}{|c|c|c|}
\hline Salbutamol MDI & Fesema (21 mcg/puff) & 2-8puff c/20min $\times 3$ veces \\
\hline NBZ con salbutamol & Salbutamol $0,5 \%(1 \mathrm{cc}=5 \mathrm{mg})$ & $\begin{array}{l}<20 \mathrm{~kg} 2,5 \mathrm{mg} / \text { dosis } \\
>20 \mathrm{~kg} 5 \mathrm{mg} / \text { dosis }\end{array}$ \\
\hline NBZ salbutamol + BI & $\begin{array}{l}\text { Salbutamol } 0,5 \% \\
\quad(1 \mathrm{cc}=5 \mathrm{mg}) \\
\text { Atrovent } 0,0025 \% \\
(1 \mathrm{cc}=0,25 \mathrm{mg})\end{array}$ & $\begin{array}{c}\text { Salbutamol: } \\
<20 \mathrm{~kg} 2,5 \mathrm{mg} / \text { dosis }>20 \mathrm{~kg} 5 \mathrm{mg} / \text { dosis } \\
\mathrm{BI}:<10 \mathrm{~kg} 250 \mathrm{mcg} / \text { dosis } \\
>10 \mathrm{~kg} 500 \mathrm{mcg} / \text { dosis }\end{array}$ \\
\hline $\begin{array}{l}\text { NBZ continua con } \\
\text { salbutamol }\end{array}$ & Salbutamol $0,5 \%(1 \mathrm{cc}=5 \mathrm{mg})$ & $\begin{array}{l}0,25-0,5 \mathrm{mg} / \mathrm{kg} / \mathrm{h}(0,05-0,1 \mathrm{ml}) \\
\text { máximo } 10 \mathrm{mg} / \mathrm{h}\end{array}$ \\
\hline $\begin{array}{l}\text { NBZ continua con } \\
\text { salbutamol }+\mathrm{Bl}\end{array}$ & $\begin{array}{l}\text { Salbutamol } 0,5 \% \\
\quad(1 \mathrm{cc}=5 \mathrm{mg}) \\
\text { Atrovent } 0,0025 \% \\
\quad(1 \mathrm{cc}=0,25 \mathrm{mg})\end{array}$ & $\begin{array}{c}\text { Salbutamol: } \\
0,25-0,5 \mathrm{mg} / \mathrm{kg} / \mathrm{hr}(0,05-0,1 \mathrm{ml}) \\
\text { BI: } 0,25-0,5 \mathrm{mg} / \mathrm{h}(1-2 \mathrm{cc} / \mathrm{h}) \\
\text { Completar volumen de } 72 \mathrm{cc} \text { de SF } 0,9 \% \\
\text { NBZ } 18 \mathrm{cc} / \mathrm{h} \times 4 \mathrm{hrs}\end{array}$ \\
\hline Prednisona & Prednisona $(20 \mathrm{mg} / 5 \mathrm{ml})$ o $(1 \mathrm{mg} / 1 \mathrm{ml})$ & 1-2mg/kg/día máximo $40 \mathrm{mg} /$ día \\
\hline Hidrocortisona & & $\begin{array}{c}10 \mathrm{mg} / \mathrm{kg} / \mathrm{día} \text { de carga, luego } 5 \mathrm{mg} / \mathrm{kg} \text { c/6hrs } \\
\text { (máximo } 100 \mathrm{mg} / \text { día) }\end{array}$ \\
\hline Metilprednisolona & & $\begin{array}{c}2 \mathrm{mg} / \mathrm{kg} \text { carga, luego } 0,5 \mathrm{mg} / \mathrm{kg} \mathrm{c} / 6 \mathrm{hrs} \\
\text { Máxima } 60 \mathrm{mg} / \mathrm{dosis} \text { carga } \\
\text { Máxima } 120 \mathrm{mg} / \text { día mantención }\end{array}$ \\
\hline Sulfato de Magnesio & $\begin{array}{l}\text { Sulfato magnesio } 25 \% \\
\qquad(1 \mathrm{cc}=250 \mathrm{mg})\end{array}$ & $\begin{array}{l}25-50 \mathrm{mg} / \mathrm{kg} / \mathrm{dosis} \text { en } 30 \text { minutos } \\
\text { (diluído en SF) (máximo } 2 \mathrm{gr} \text { ) }\end{array}$ \\
\hline
\end{tabular}

Bl: bromuro de ipatropio, SF: solución fisiológica. MDI: inhalador presurizado de dosis medida. NBZ: Nebulización

según puntaje obtenido, en leve (puntaje 5-7), moderada (811) y severa (12-15) al ingreso al hospital y a los 30, 60 y 120 minutos (18).

Las evaluaciones e intervenciones fueron llevadas a cabo según un algoritmo preestablecido basado en el PAS (Figura 1) por los residentes de pediatría, y se adjuntó a la ficha clínica de cada paciente una lista de verificación donde se detalló el tratamiento según la categorización del paciente en los tiempos definidos.

Los criterios de hospitalización fueron la persistencia de requerimiento de oxigenoterapia pese a la primera línea de tratamiento en el servicio de urgencia (B2, corticoides con o $\sin \mathrm{BI}$ ). Los criterios de ingreso a intermedio fueron requerimiento de AVNI y falla de tratamiento con OTAF y sulfato de magnesio. Los criterios de ingreso a intensivo fueron insuficiencia respiratoria aguda que no mejora con AVNI (Sa/FIO2 < 180), insuficiencia ventilatoria con $\mathrm{pH}<$ $7,2 \mathrm{PaC0} 2>60 \mathrm{mmhg}$, requerimiento de broncodilatadores endovenosos, inestabilidad hemodinámica, indicación de intubación para ventilación mecánica invasiva o manejo de complicaciones severas (neumotórax, atelectasia masiva, infección respiratoria aguda grave).

Los pacientes ingresados al servicio de pediatría con puntaje clínico leve, se manejaron con broncodilatadores beta 2 agonista en inhalador de dosis medida (IDM) y corticoides orales (prednisona), salvo que hubiesen recibido previamente corticoides endovenosos en el servicio de urgencia.
Aquellos con puntaje clínico moderado o con deterioro clínico en el transcurso de la hospitalización, recibieron nebulizaciones de salbutamol asociado a BI cada 20 minutos durante 1 hora, corticoides endovenosos de preferencia metilprednisolona en dosis habituales, usando carga, o dosis de mantención en caso de haber sido utilizados previamente en el servicio de urgencia. Si en el servicio de urgencia se inició otro corticoide endovenoso como hidrocortisona, éste fue continuado en dosis de mantención. La presentación y dosis de los fármacos utilizados se detalla en la Tabla 2. Estos pacientes, que correspondieron al grupo con puntaje $>8, \mathrm{Sp} 02<90 \%$ con FiO2 0,35; Sa/FiO2< 260, ingresaron a unidad de cuidados medios (mediana complejidad) donde se inició oxigenoterapia de alto flujo (OTAF) según criterios preestablecidos en la Tabla 3 y terapia de segunda línea con sulfato de magnesio endovenoso en dosis de $50 \mathrm{mg} / \mathrm{kg}$ en 30 minutos. En caso de falta de respuesta clínica durante 1 hora, manifestada por una Sp02 < 90\% con FiO2 > 0.4, Sa/FiO2 < 260, como en aquellos que ingresaron desde el SU con puntaje clínico severo, se procedió a trasladar a la unidad de cuidados intermedios para la instalación de asistencia ventilatoria no invasiva (AVNI) (Tabla 3) según criterios preestablecidos. En ausencia de mejoría clínica se decidió traslado a unidad de cuidados intensivos.

Para la administración de la Oxigenoterapia de alto flujo se utilizaron equipos Airvo de Fisher Pykel, los que entregan en el mismo dispositivo la termohumedificación con 
Tabla 3. Criterios y parámetros de indicación de OTAF y AVNI.

\begin{tabular}{|c|c|c|}
\hline & OTAF & AVNI \\
\hline Criterios & $\begin{array}{c}\text { Score }>8 \\
\text { SpO2 }<90 \% \text { con FiO2 0,35 } \\
(\text { Sa/FiO2 }<260)\end{array}$ & $\begin{array}{c}\text { Score }>8 \text {, falla } 1 \text { h OTAF } \\
\text { Sp02 }<90 \% \text { con FiO2 }>0,40 \\
(\text { Sa/FiO2 }<260)\end{array}$ \\
\hline Parámetros & $\begin{array}{c}\text { Para el niño mayor de } 2 \text { años: } \\
1 \mathrm{lt} / \mathrm{kg} / \mathrm{min} ; 20-50 \text { LPM }\end{array}$ & $\begin{array}{l}\text { I/E: } 10 / 6 \mathrm{cmH} 20 \text { hasta } 20 / 8 \\
\text { Ti: } 0,8-1,2 \text { FR: } 12-20 \text { RPM }\end{array}$ \\
\hline
\end{tabular}

OTAF: Oxigenoterapia de alto flujo con cánula nasal. AVNI: asistencia ventilatoria no invasiva.

LPM: Flujo expresado en litros por minuto. Sp02: Saturometría de oxígeno, no invasiva.

Fi02: Fracción inspirada de oxígeno. I: presión inspiratoria, E: presión espiratoria.

Ti: tiempo inspiratorio. FR: frecuencia respiratoria

una base de cascada más la generación de flujos controlados a través de una turbina. Permiten la lectura de la Fi02 obtenida mezclando oxigeno desde la red central a través de un flujómetro balanceado con el flujo de aire seleccionado. La interfase de conexión al paciente es una naricera ad hoc que admite flujos hasta 50 LPM, sin generar resistencia, la tubuladura o circuito de conexión es de $15 \mathrm{~mm}$ de diámetro y tiene un sistema calefactor endomural. Se utilizaron flujos de 1 LPM por kilo de peso, con flujo máximo de 30 LPM y una temperatura de termohumedificación de 34 grados $C^{\circ}$.

La asistencia ventilatoria no invasiva (AVNI) se entregó a través de generadores de flujos híbridos Trilogy 202 de Philips, que permiten entregar una FiO2 conocida a través de un blender. La FiO2 utilizada fue la necesaria para mantener una Spo2 entre 93 y 95\%, las modalidades de ventilación fueron $\mathrm{S} / \mathrm{T}$ con presión en binivel, utilizando la FR elegida según la edad del paciente, una presión positiva espiratoria (Epap) mínima de 6 a $8 \mathrm{~cm}$ de $\mathrm{H} 20$ y una presión positiva inspiratoria (Ipap) de 10 a $20 \mathrm{~cm}$ de $\mathrm{H} 20$, que permitiera una presión diferencial de al menos $4 \mathrm{~cm}$, con un tiempo de transición (Risetime) de Epap a Ipap rápido de 100 ms. La Ipap se ajustó subiendo de a $2 \mathrm{~cm}$ de $\mathrm{H} 20$ cada 30 minutos, buscando la disminución del trabajo respiratorio (disminución de la FR y FC, retracción y/o tiraje supraesternal, ausencia de respiración paradojal y mejoría de la Sp02).

Según la adaptación del paciente se utilizaron como interfases mascarillas total face de Philips o nasales Philips o Fisher Pykel, con tallas según peso y forma de la cara que permitiera el menor flujo de escape posible. No se usó sedación (13).

La terapia broncodilatadora en los pacientes con AVNI, durante el periodo con uso de algoritmo se realizó con nebulizadores de malla Aerogen unidos al brazo seco de la base de termohumedificación Fisher Pykel MR810. Este nebulizador de malla genera partículas de 2 ug de diámetro medio aerodinámico y utiliza un generador eléctrico o procesador que permite entregar NBZ continua de $12 \mathrm{ml} /$ hora o discontinua de broncodilatadores. Para la terapia broncodilatadora con inhalador de dosis medida (IDM) se usó salbutamol, Fesema de GlaxoSmithKline, con aerocámara estándar no valvulada de 350 - $500 \mathrm{ml}$. La OTAF no fue suspendida al momento de realizar la maniobra de inhalación. En la cohorte del año 2016, la terapia broncodilatadora en los pacientes en AVNI se realizó con IDM y adaptador proximal al circuito de ventilación utilizando 4 disparos (puff) del IDM y la nebulización continua se realizó con un circuito en T y un nebulizador Hudson Draft II con un volumen de $4 \mathrm{ml}$ y débito de $18 \mathrm{ml}$ por hora.

Para la monitorización se utilizaron monitores cardiorespiratorios Mindray en la unidad de intermedio y monitores de signos vitales Mindray o General Electric en las unidades de internación de complejidad media. Ambos tienen tecnología de lectura Nellcor. No hubo monitorización de Co2 espirado (ETCO2). Sólo se consideró realizar gases en sangre en aquellos pacientes con insuficiencia respiratoria aguda grave con mala respuesta clínica a la AVNI, que mantuvieron requerimiento de Fi02 sobre 0.5 y Sp02 < de 90\% (Sa/FiO2 $<180$ ), con potencial indicación de intubación.

Los criterios de alta fueron: puntaje de severidad en categoría leve y saturación > 0 igual a 93\% por 12 horas sin oxigenoterapia.

Para evaluar el impacto del algoritmo de tratamiento, incluyendo la OTAF, el sulfato de magnesio endovenoso en etapas más precoces y el uso de nebulizador de malla para la entrega de broncodilatadores en pacientes en AVNI, se midieron dos variables primarias: la caída de 2 puntos en el puntaje clínico utilizado al finalizar el algoritmo, considerada como clínicamente significativa en un estudio reciente (19) y la disminución en los días de hospitalización en comparación con los pacientes tratados durante la cohorte del año 2016, sin algoritmo. Como variable secundaria de eficacia se evaluó la necesidad de traslado a cama crítica: unidad de cuidados intermedios o unidad de cuidados intensivos.

\section{ANÁLISIS ESTADÍSTICO}

El análisis estadístico se realizó mediante el programa STATA 15. Las variables cuantitativas fueron expresadas como mediana y rango, y las variables cualitativas fueron expresadas en frecuencia y porcentaje. Para la comparación de los 2 grupos se utilizó como prueba no paramétrica el test de Mann Whitney. Para la comparación de PAS en su evolución dentro de los 120 minutos de la aplicación del algoritmo se utilizó test de Wilcoxon. Se consideró estadísticamente significativo $p<0,05$.

Este estudio fue aprobado por el Comité de Ética del 
Servicio de Salud Metropolitano Central (Resolución n 1303) y se contó con el consentimiento informado de los padres y asentimiento de aquellos pacientes mayores de 7 años.

\section{RESULTADOS}

En este estudio se incluyó un total de 106 pacientes ingresados al Servicio de Pediatría del HCSBA con diagnóstico de asma agudo, 55 pacientes durante el período de aplicación del algoritmo entre diciembre del 2016 a diciembre del 2017 y 51 pacientes del grupo control sin aplicación del algoritmo, ingresados durante el año 2016.

Ambos grupos fueron similares y comparables en cuanto a edad, sexo, diagnóstico previo de asma, tabaquismo en los padres y número de exacerbaciones que requirieron consultas en servicio de urgencia y hospitalizaciones en el año anterior. Hubo diferencia no estadísticamente significativa en el estado nutricional, con mayor frecuencia de sobrepeso en grupo sin algoritmo, en el límite de la significancia, 13(25,5\%) vs $7(12,7 \%) \quad p=0.057$. Hubo diferencias significativas en rinitis alérgica y uso de tratamiento de mantención del asma en el grupo sin algoritmo, 39 (76.5\%) vs $19(34,5 \%)$ p < $0.001 ; 36(70,5 \%)$ vs $25(45,5 \%) p<0.001$ respectivamente (Tabla 4).

Al ingreso al servicio de pediatría 10 (18,2\%) pacientes tuvieron categoría de asma agudo leve; moderado $40(72,7 \%)$ y severo $5(9,1 \%)$. La mediana del puntaje de severidad fue 8 (p.50, IQR 8-10). En el control realizado a los 30 minutos del ingreso, la mediana del puntaje de severidad fue 6 (p.50, IQR 6-10) con una caída significativa en el puntaje clínico de 2 puntos $(p<0.001)$.

Al control de los 60 minutos se mantuvo la mediana de puntaje de severidad en 6 . A los 120 minutos la mediana de puntaje se severidad bajó a 5 (p.50, IQR 5-7), observando una caída total en el puntaje clínico de 3 puntos luego de la aplicación del algoritmo ( $p<0.001)$. (Figura 2$)$

Tabla 4. Comparación de las características demográficas y basales de los 2 grupos estudiados.

\begin{tabular}{|c|c|c|c|}
\hline Variables & Año 2016 & Año 2017 & $P$ value \\
\hline & Sin algoritmo $(n=51)$ & Con algoritmo $(n=55)$ & \\
\hline Edad años, mediana (rango) & $8,05(5-14)$ & $8,2(5-14)$ & ns \\
\hline \multicolumn{4}{|l|}{ Sexo $(\%)$} \\
\hline Femenino & $27(52,9)$ & $23(41,8)$ & ns \\
\hline Masculino & $24(47)$ & $32(58,1)$ & ns \\
\hline Diagnóstico previo asma, $\mathrm{n}(\%)$ & $36(70,5)$ & $37(67,27)$ & ns \\
\hline Tratamiento mantención, $\mathrm{n}(\%)^{*}$ & $36(70,5)$ & $25(45,45)$ & $P<0,001$ \\
\hline \multicolumn{4}{|l|}{ Estado nutricional, $\mathrm{n}(\%)$} \\
\hline Eutrófico & $14(27,45)$ & $34(61,81)$ & ns \\
\hline Sobrepeso & $13(25,49)$ & $7(12,72)$ & p 0,057 \\
\hline Obeso & $15(29,41)$ & $11(20)$ & ns \\
\hline Riesgo desnutrición & 3 & 1 & \\
\hline Sin registro & 6 & 2 & \\
\hline Tabaquismo en los padres, $\mathrm{n}(\%)$ & $30(58,82)$ & $31(56,36)$ & ns \\
\hline Antecedentes de rinitis, $\mathrm{n}(\%)$ & $39(76,47)$ & $19(34,54)$ & $p<0,001$ \\
\hline $\begin{array}{c}\mathrm{N}^{\circ} \text { consultas previas SU, mediana } \\
\text { (rango) }\end{array}$ & $1,41(0-6)$ & $0,78(0-4)$ & ns \\
\hline $\begin{array}{c}\mathrm{N}^{\circ} \text { hospitalizaciones previas, } \\
\text { mediana (rango) }\end{array}$ & $0,19(0-1)$ & $0,21(0-2)$ & ns \\
\hline
\end{tabular}

Test de Mann Whitney

ns: no significativo, SU: servivio de urgencia

* Corticoide inhalado (budesonida 400ug/dia o Fluticasona 250 a 500 ug/dia) o corticoide inhalado + B2 de accion prolongada (Fluticasona+Salmeterol 250/25 ug/dia). Ninguno con tratamiento regular para rinitis alérgica. 
Figura 2. Caída de Puntaje clínico y Disminución días de Hospitalización.

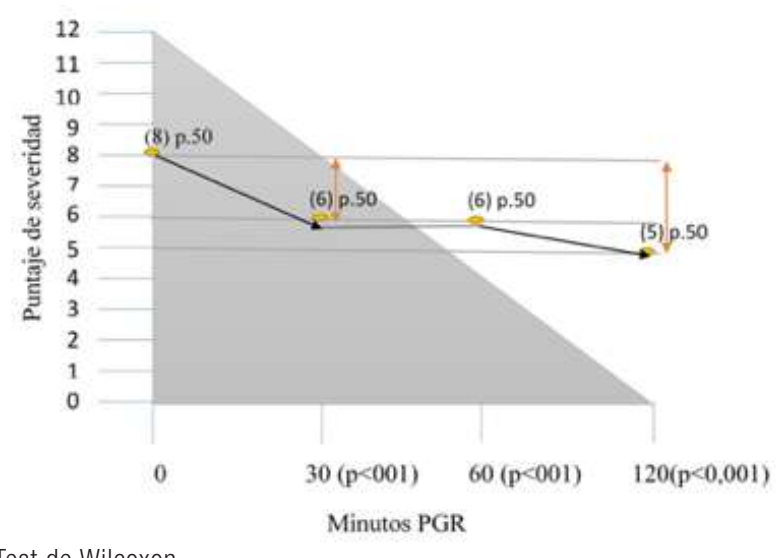

Test de Wilcoxon

Un 30.9\% de los niños en que se les aplicó el algoritmo, requirieron OTAF, lo que no fue utilizado en el grupo control.

En ambos grupos no hubo diferencias significativas para las hospitalizaciones en intermedio. Cerca de un 20\% de los pacientes fueron ingresados a intermedio para recibir AVNI.

La duración de la oxigenoterapia fue similar, con una media de 3,6 días. La Fi02 máxima no tuvo diferencias, con un rango de 0,3 a 0,5.

No hubo diferencias en la frecuencia de uso de sulfato de magnesio endovenoso, $4(7,3 \%)$ y $3(5,9 \%)$ en el grupo con y sin algoritmo respectivamente.

En el grupo con algoritmo hubo una diferencia no significativa de los días de hospitalización en intermedio y significativa en la duración total de la internación: 0,6 días y 0,95 días respectivamente $(p<0,0368)$ pasando de 3,5 días (0-8) a 2,9 días (0-7) en hospitalización en intermedio y 4,78 días (2-17) a 3,83 (1-12) en duración total de la internación. Complicaciones caracterizadas por atelectasias ocurrieron en 5 pacientes (9\%) del grupo del estudio y 3 pacientes $(5,88 \%)$ en el grupo control (diferencia no significativa). La neumonía fue significativamente más frecuente en el grupo no sometido al algoritmo $(24(43,6 \%)$ vs $3(5,9 \%) p<0.001)$.

Se obtuvo confirmación de un agente infeccioso con Inmunofluorescencia viral, y con técnica de PCR (Film array; para quienes requirieron AVNI) en $17(30,9 \%)$ y $11(21,56 \%)$ pacientes del grupo de estudio y control respectivamente. La etiología viral fue la más frecuente. Otras etiologias fueron Mycoplasma Pneumoniae y Chlamydia Pneumoniae.

Ningún paciente requirió traslado a unidad de tratamiento invasivo, intubación para ventilación mecánica, ni presentó otras complicaciones como neumotórax u otras modalidades de escape aéreo. No hubo letalidad.

\section{DISCUSIÓN}

El objetivo de este estudio fue describir y evaluar el impacto de un algoritmo de tratamiento en niños hospitalizados por crisis de asma refractaria al tratamiento de primera línea en el servicio de urgencia, aplicando un plan de mejoramiento continuo que incorporase elementos clínicos en la toma priorizada de decisiones para el tratamiento del asma agudo. Es así como de acuerdo al mejor nivel de evidencia disponible, se planteó como hipótesis que el uso de un protocolo preestablecido de manejo escalonado en tiempos definidos, junto con la utilización de otros dispositivos como la OTAF y nebulizadores de malla y el uso precoz de sulfato de magnesio, aplicado en pacientes categorizados con un puntaje clínico de asma agudo, generaría una mejoría clínica precoz, disminución en los días de uso de cama crítica y en los días totales de hospitalización en comparación al tratamiento habitual utilizado previamente.

El tratamiento escalonado en asma agudo pediátrico está bien definido por líneas y etapas, respondiendo un $80 \%$ de los pacientes a terapias de primera línea. El 20\% restante, que no responde, se cataloga como asma refractaria (9) y en ellos se implementó el algoritmo basado en un puntaje clínico.

Se consideró como terapia de primera línea el uso de broncodilatadores en inhaladores de dosis medida (IDM) y de corticoides sistémicos. Existe información disponible que el uso de broncodilatadores IDM otorga cierta ventaja frente al uso de nebulizaciones, incluso en exacerbaciones asmáticas de mayor severidad (10). Otro tratamiento de primera línea en crisis moderadas y severas es la asociación de un beta 2 agonista con bromuro de ipratropio en dosis repetidas, demostrándose efectividad en reducir la frecuencia de hospitalización, sin embargo, no se ha demostrado el beneficio de esta asociación en el manejo del paciente hospitalizado (4).

Las herramientas de evaluación para clasificar y priorizar a los pacientes con una crisis aguda de asma son útiles para su manejo, brindando la mejor atención en cada situación.

Pese a que existen diversos puntajes clínicos con el fin de categorizar la severidad del asma no existe consenso respecto a cuál utilizar. El Pulmonary Score (17) ha sido incorporado recientemente para predecir el riesgo de hospitalización, necesidad de unidad de cuidado intensivo (19), y para valorar los resultados de la utilización de OTAF (12). En nuestro estudio se utilizó el Pediatric Asthma Score (PAS), ya que los puntos de corte de Sp02, facilitan la valoración no invasiva de la severidad de la insuficiencia respiratoria en pacientes con requerimientos de oxigenoterapia.

Observamos que en el período en que se incorporó el algoritmo hubo una rápida disminución en el puntaje clínico de severidad a los 120 minutos y una disminución significativa de los días totales de hospitalización respecto al grupo control. Este efecto puede ser atribuido no sólo a la aplicación del algoritmo, sino que también al uso precoz de sulfato de magnesio, al uso de OTAF y de nebulizadores de malla.

Se ha demostrado que el sulfato de magnesio endovenoso disminuye la progresión de una exacerbación asmática a una insuficiencia respiratoria grave $(20,21,22)$. Como terapia de tercera línea utilizamos tanto OTAF 
Tabla 5. Comparación de los resultados del tratamiento de la crisis de asma en ambos grupos estudiados.

\begin{tabular}{|c|c|c|c|}
\hline Variables & Año 2016 & Año 2017 & $P$ value \\
\hline & Sin algoritmo $(n=51)$ & Con algoritmo $(n=55)$ & \\
\hline Ingreso a intermedio $\mathrm{n}(\%)$ & $9(17,6)$ & $10(18,2)$ & ns \\
\hline Ingreso a UCIP n(\%) & 0 & 0 & \\
\hline Mortalidad n(\%) & 0 & 0 & \\
\hline Neumotórax $\mathrm{n}(\%)$ & 0 & 0 & \\
\hline Atelectasia $\mathrm{n}(\%)$ & $3(5,88)$ & $5(9)$ & ns \\
\hline Neumonía n(\%) & $3(5,9)$ & $24(43,6)$ & $<0.001$ \\
\hline Días total de 02 ,media (rango) & $3,6(1-13)$ & $3,6(1-10)$ & ns \\
\hline Días totales de Hospitalización & $4,78(2-17)$ & $3,83(1-12)$ & $<0.05$ \\
\hline Días totales en Intermedio & $3,5(0-8)$ & $2,9(0-7)$ & ns \\
\hline Fi02 Máxima, media (rango) & $0,4(0,3-0,5)$ & $0,37(0,3-0,5)$ & ns \\
\hline OTAF n(\%) & 0 & $17(30,9)$ & na \\
\hline AVNI n(\%) & $9(17,6)$ & $10(18,2)$ & ns \\
\hline Magnesio $\mathrm{n}(\%)$ & $3(5.9)$ & $4(7,3)$ & ns \\
\hline \multicolumn{4}{|l|}{ Comorbilidad Infecciosa n(\%) } \\
\hline VRS*1 & $4(7,84)$ & $4(7,27)$ & ns \\
\hline Otros virus (VIA, MPV,PI)*1 & $5(9,80)$ & $4(7,27)$ & ns \\
\hline Rinovirus*2 & na & $6(12,72)$ & na \\
\hline Mycoplasma*3 & $2(3,92)$ & $2(3,6)$ & na \\
\hline Otro Agente (Clamydia Pneumoniae)*4 & 0 & $1(1,81)$ & ns \\
\hline Total Etiología (+) & $11(21,56)$ & $17(30,9)$ & ns \\
\hline Puntaje leve (PAS) & na & $10(18,2)$ & na \\
\hline Puntaje moderado (PAS) & na & $40(72,7)$ & na \\
\hline Puntaje severo (PAS) & na & $5(9,1)$ & na \\
\hline
\end{tabular}

Test de Mann Whitney

ns: no significativo, na: no aplica.

*1 IFV (Inmunofluorescencia viral) ó Film Array (año 2017) / *2 Film Array (PCR multiple) / *3 PCR (Reaccion cadena polimerasa específica) *4 IgM (Anticuerpo específico) / Fi02: Fracción inspirada de oxigeno OTAF: Oxigenoterapia de alto flujo AVNI: asistencia ventilatoria no invasiva VRS: virus respiratorio sincicial. VIA: virus influenza A. MPV: metaneumovirus, PI: virus parainfluenza. PAS: Pedatric Asthma Score.

como AVNI. La oxigenoterapia de alto flujo (OTAF) podría ser usada en la actualidad, dentro del manejo escalonado del asma agudo, como terapia complementaria y mejor tolerada que la AVNI, como ha sido reportado en estudios observacionales hechos en adultos con insuficiencia respiratoria aguda (23). La creciente disponibilidad de dispositivos en unidades de cuidados intensivos pediátricos, y más recientemente en sala de pediatría, así como su facilidad de uso y tolerancia, ha llevado a su incorporación en el manejo de niños con insuficiencia respiratoria (24). En un estudio retrospectivo/ observacional de niños de 4 a 15 años con diagnóstico de crisis aguda de asma moderada a severa se compararon aquellos tratados con oxigenoterapia convencional $\mathrm{v} / \mathrm{s}$ OTAF, encontrándose que el uso de esta última modalidad produjo una reducción en la frecuencia cardiaca, frecuencia respiratoria y en el puntaje clínico de asma en las primeras 3 a 6 horas del tratamiento. Se demostró que aquellos pacientes que iniciaron OTAF con un flujo $>15 \mathrm{lt} / \mathrm{min}$ ingresaban menos a la UCIP que los que iniciaron con un flujo $<15 \mathrm{lt} / \mathrm{min}(13 \%$ $\mathrm{v} / \mathrm{s} 47 \%, \mathrm{P}=0,05)(25)$.

Si bien existen pocos estudios que avalen la eficacia y utilidad de OTAF en crisis aguda de asma en niños, se ha observado mejoría en parámetros intermedios y en puntajes clínicos que expresan la disminución de la dificultad respiratoria y la mejoría en la Sp02. Esto se atribuye a una mejor termohumedificación, mayor aclaramiento mucociliar, lavado del espacio muerto nasofaríngeo y en la entrega de oxígeno más estable, produciendo una presión positiva en la vía aérea al final de la espiración variable, que se ha demostrado ser más útil en los pacientes con bronquiolitis (26).

La asistencia ventilatoria no invasiva es eficaz como tratamiento de tercera línea en pacientes con crisis asmática severa. El mecanismo de acción parece estar basado en 
un efecto broncodilatador directo, en el mejoramiento del reclutamiento alveolar y en un aumento en la respuesta a Ios broncodilatadores $(27,28)$. Sin embargo, la guías clínica GINA 2019 no considera el uso de AVNI como parte de las intervenciones convencionales en el asma, ya que el nivel de evidencia de su uso en crisis es escaso. Las últimas guías GINA recomiendan que el uso de AVNI debe ser en pacientes vigilados y no debe intentarse en pacientes agitados, ni estos deben ser sedados para su uso (15).

Dado el beneficio observado para estos nuevos tratamientos y terapias respiratorias, se decidió aplicar el algoritmo que incorpora la toma de decisiones según la severidad y respuesta en etapas escalonadas, agregando al tratamiento convencional, del período previo, el uso de sulfato de magnesio endovenoso precoz, OTAF y AVNI sin sedación en planta pediátrica y unidad de cuidado intermedio. Estas medidas permitieron una disminución de los días de hospitalización. Lo que permitió mayor rotación de camas, I0 que es de gran importancia en los períodos de alta demanda epidemiológica.

\section{LIMITACIONES}

La principal limitación del estudio fue no tener un grupo control para comparar el algoritmo con un grupo no intervenido, en que el tratamiento se hubiese guiado según el estándar del período previo. Este hecho debilita el poder establecer conclusiones de efectividad y eficiencia. Por otro lado, la disminución de la hospitalización comparada con los pacientes no sometidos al algoritmo, puede deberse a múltiples factores y no necesariamente a las intervenciones realizadas los primeros 120 minutos. No obstante, dado que hubo una frecuencia similar de AVNI y de utilización de sulfato de magnesio endovenoso es posible que la incorporación de OTAF en 1/3 de los pacientes ingresados al algoritmo, pudiese explicar estos resultados.

No se pudo contar con un grupo control paralelo al grupo en el que se uso el algoritmo, ya que la aplicación de este último fue una política de mejoramiento continuo y buenas prácticas, en que se estimó que existían evidencias que justificaban, que la totalidad de los pacientes con asma agudo, fueran incluidos en el algoritmo.

Por este motivo, incluimos otras variables de efectividad, como fue la duración de la hospitalización y utilización de recursos de cama crítica que permitieran las comparaciones con un grupo demográficamente similar que no hubiese utilizado algoritmo en el periodo previo a su implementación. No pudimos comparar los beneficios de utilizar OTAF en asma agudo, ya que esta modalidad terapéutica se incorporó a nuestro hospital a partir del año 2017.

No realizar pruebas de validación del puntaje clínico utilizado es una limitación, pero se realizó una capacitación previa a todos los residentes, y también a la residencia de enfermería, siendo responsabilidad de los primeros la evaluación del puntaje clínico para la toma de las decisiones. Pese a que ambos grupos de pacientes tenían una frecuencia similar de diagnóstico previo de asma, solo el $25 \%$ de los pacientes incorporados al algoritmo estaban recibiendo tratamiento controlador, coincidente con lo reportado en el estudio multicéntrico de Herrera et al, en el que sólo el $20 \%$ de los niños hospitalizados por crisis de asma tenían tratamiento controlador (14).

Para la comparación de los días de hospitalización, las características demográficas y basales de los 2 grupos parecen estar balanceadas, pero los pacientes sin algoritmo podrían tener un fenotipo de mayor severidad relacionado a sobrepeso y rinitis alérgica.

Sin embargo, como ya se mencionó, el grupo no sometido al algoritmo tenía una frecuencia significativamente mayor de tratamiento controlador del asma y significativamente menor de neumonía. Sin diferencias significativas en la confirmación de la causa etiológica. Pese a que esto pudiese impactar en el control del asma y en la presencia de gatillantes vinculados a una exacerbación asmática de mayor severidad como son los virus (29), ambos grupos no tuvieron diferencias en los episodios de agudizaciones mayores como son consultas en SU y hospitalizaciones previas, sugiriendo un nivel de control del asma similar.

Ambos grupos accedieron a la misma medicación controladora, que es la disponible en la farmacia de especialidades de nuestro hospital, vinculada a corticoides inhalados o corticoides inhalados asociados a B2 de acción prolongada y ocasionalmente montelukast.

Otra importante limitación fue no implementar el algoritmo desde el momento de ingreso al servicio de urgencia infantil, a lo menos para la incorporación de una segunda línea de tratamiento con sulfato de magnesio. La reciente experiencia en que se observó disminución en las hospitalizaciones, costos totales y duración de la hospitalización en pacientes tratados con infusión endovenosa de dosis alta e sulfato de magnesio, la hace una estrategia muy interesante (21). Pero dado que este tratamiento de segunda línea y otros manejos avanzados de la insuficiencia respiratoria aguda como son la oxigenoterapia de alto flujo y asistencia ventilatoria no invasiva no son acciones que estén implementadas rutinariamente en el servicio de urgencia de nuestro hospital, nos pareció prudente en una etapa inicial, continuar el manejo de los niños con asma aguda refractarios al tratamiento de primera línea en la planta pediátrica.

\section{CONCLUSIÓN}

El uso de un algoritmo nos permitió unificar criterios, para implementar un flujograma de tratamiento, que garantice realizar acciones tiempo dependientes, con un buen respaldo de evidencia. Se observó que el algoritmo y la aplicación de OTAF y sulfato de magnesio precoz, tuvieron una rápida disminución del puntaje clínico utilizado y que la duración de la hospitalización fue menor comparado con el periodo previo sin estas medidas. Nuestros resultados podrían sugerir que la aplicación de un algoritmo basado en puntaj clínico, junto con la incorporación de OTAF y sulfato de magnesio precoz, puede mejorar la efectividad y eficiencia del tratamiento del asma agudo. Sin embargo, se requieren hacer validaciones a través de estudios controlados. 
Los autores declaran no tener conflictos de interés.

Agradecimientos: a las residencias de enfermería y pediatría del Hospital Clínico San Borja Arriarán, por el interés y apoyo para la implementación del protocolo guiado por resultado en el Asma agudo y por mantenerlo vivo después de la finalización de este trabajo.

\section{REFERENCIAS}

1. The Global Asthma Report 2018. http://www. globalasthmareport. org/burden. ISBN: 978-0-47346524-7 (ELECTRONIC)

2. Mallol J., Cortez E., Amarales L., et al. Prevalencia del asma en escolares chilenos. Estudio descriptivo de 24.470 niños. ISAAC-CHILE. Rev Med Chile 2000; 128: 279-85

3. Mallol J., Aguirre V., Aguilar P.; et al. Changes in the prevalence of asthma in chilean school age children between 1994 and 2002. International study of asthma and allergies in childhood (ISAAC), Chile phase I and III. Rev Med Chil. 2007; 135:580-6.

4. Mintegui S., Fernández J., González-Balenciaga M., et al. Consequences of strength treatment of children with asthma in a pediatric emergency department on to inhospital admission. Emergencias 2003; 15:345-50.

5. Fernández J., Mintegui S., Sánchez J., et al. Recent changes in emergency room visits and hospitalization for asthma in children. An Esp Pediatr 1998; 49:577-81.

6. Newth C., Meert K., Clark A., et al. Fatal and near-fatal asthma in chidren: the critical care perspective. J Pediatr 2012; 161: 214-21.

7. Verscheure F. Estado asmático en Pediatría. Neumol Pediatr. 2016; 11:155-161.

8. Indinnimeo L., Chiappini E., Miraglia M. Guideline in management of the acute asthma in children by Italian Sociaty of Pediatrics. Italian Journal of Pediatrics. 2018;44:2-10

9. Pardue B., Fleming G., Kaye J., et al. Pediatric acute asthma exacerbations: Evolutions and management from emergency department to intensive care unit. Pediatric Asthma. 2016; 53:607-617.

10. Kelly C., Andersen C., Pestian J., et al. Improved outcomes for hospitalized asthmatic children using a clinical pathway. Ann Allergy Asthma Immunol. 2000; 84:509-16.

11. Liu X., Yu T., Rower J., et al. Optimizing the use of intravenous magnesium sulfate for acute asthma treatment in children. Pediatr Pulmonol. 2016; 51:14141421.

12. Ballesteros Y., De Pedro J., Portillo N.,et al. Pilot Clinal Trial of High-Flow Oxygen Therapy in Children with Asthma in the Emergency Service. J Pediatr. 2018; 194:204-210.

13. Parga D., Zambrano H., Valdebenito C., et al. Ventilación mecánica no invasiva en el manejo del estado asmático en pediatría. Arch Pediatr Urug 2017; 88:284-287.

14. Herrera A. Hospitalizations for asthma exacerbations in Chilean Children: A multicenter observational study. Allergol Inmunophatol. 2018; 46: 533-538.

15. Global Initiative for asthma. Global strategy for asthma management and prevention, 2019. Available from: www. ginasthma.org

16. Fernandez-Nievas F., Anand K. Severe acute asthma exacerbation in children: a stepwise approach for escalating therapy in the pediatric intensive care unit. $J$ Pediatr Pharmacol Ther 2013; 18(2): 88-104.

17. Smith S., Baty J., Hodge D. Validation on the pulmonary score: an asthma severity score for children. Acad Emerg Med. 2002; 9:99-104.

18. Babaic N., Valdebenito C., Koppmann A., et al. Asma Agudo Pediatrico. El desafío del manejo escalonado. Neumol Pediatr. 2017; 12:114-121.

19. Panigua N., Elosegi A., Duo I., et al. Initial Asthma Severity Assessment Tools as Predictors of Hospitalizations. Journal of Emergency Medicine. 2017; 53:10-17.

20. Ohn M., Jacob S. Magnesium should be given to all children presenting to hospital with acute severe asthma. Pediatr Respir Rev. 2014; 15:319-32

21. Irazuzta J., Paredes F., Pavlicich V., et al. High-Dose Magnesium Sulfate Infusion for Severe Asthma en the Emergency Department: Efficacy Study. Pediatr Crit Care Med. 2016; 17:29-33.

22. Zhantao S., Rui K., Zhongtao G. Intravenous and nebulized magnesium sulfate for treating acute asthma in children: A Systematic Review and Meta-analysis.Pediatr Emerg Care. 2018; 34:390-395.

23. Frat J., Brugere B., Ragot S., et al. Sequential application of oxygen therapy via high flow nasal cannula and noninvasive ventilation in acute respiratory failure: and observational pilot study. Respir Care. 2015; 60: 170180.

24. Ingvild B., Davis P., Oymar K. High flow nasal cannula in children: a literature review. Scand J Trauma Resusc Emerg Med. 2016; 24:93.

25. González F., González M., Toledo B., et al. Tratamiento con oxigenoterapia de alto flujo en las crisis asmáticas en la planta de hospitalización de pediatría: nuestra experiencia. An Pediatr. 2019; 90:72-80.

26. Milesi C., Boubal M., Jacquot A., et al. High flow nasal cannula: recommendation for daily practice in pediatrics. Ann intensive care. 2014; 4:29-35.

27. Kristin C., Zafar A., Smith B. Noninvasive ventilation in acute severe asthma: Current evidence and future perspectives. Pulmonary Medicine. 2014; 20:118-123.

28. Konang S., Feinberg J., Wettersten J., et al. Non-invasive positive pressure ventilation for acute asthma in children. Cochrane Database Syst Rev. 2106; 9.

29. Nguyen-Thi-Dieu T., Le-Thi-Minh H., Phan-Naht A., et al. Study of Clinical Characteristics and Cytokine Profiles of Asthmatic Children with Rhinovirus Infections during Asthma Exacerbations at National Hospital of Pediatrics. Can Respir J. 2018; 2018: 9375967. 